\title{
Prevalencia de Patologías Colorectales en estudios Colonoscopicos: servicio de cirugía general - Hospital Militar Central de las Fuerzas Armadas
}

\section{RESUMEN}

Amanda Fretes

amandafretes@hotmail.com

Hernando Raúl Cardozo

raulcardozoarias@gmail.com

Alejandro Caballero mansoncaballero87@icloud.com

Ricardo Isaías Fretes ricardof@ rieder.net.py

Ingrid Melo

Introducción: La colonoscopia realiza una exploración visual de la mucosa del colon, dentro de sus objetivos está la localización de lesiones colonicas, obtener biopsias para su análisis, realizar diversos procedimientos terapéuticos o diagnosticar lesiones pequeñas no detectables por métodos imagenológicos tradicionales. El objetivo del presente estudio es determinar la prevalencia de Patologías Colorrectales y sus características en pacientes sometidos a colonoscopías en el Servicio de Cirugía General del Hospital Militar Central de las FFAA durante el periodo 2018 a 2019.

Metodología: estudio observacional, descriptivo, retrospectivo de corte transverso.

Resultados: Fueron estudiados 355 historias clínicas e informes de colonoscopias. Del total el $56 \%$ correspondieron al sexo Femenino. El promedio de edad fue de 54 años, el grupo etario más prevalente fue de 50 a 60 años. El principal motivo de indicación de colonoscopia fue Screninng de Cáncer Colorrectal, vista en 51\% de los pacientes, seguido de hemorragia digestiva baja en $27 \%$ de los pacientes. Los hallazgos más frecuentes en la colonoscopia fueron Hemorroides en $36 \%$ de los pacientes, seguido de Polipos $27 \%$ y Divertículos $18 \%$. En cuanto a la localizaciones más frecuentes: el canal anal $40 \%$, Colon sigmoides $32 \%$ y colon descendente $18 \%$. Los hallazgos histológicos, en pólipos el tipo Hiperplasico en un $43 \%$ y en tumores fue el adenocarcinoma de colon en un $82 \%$ de los casos. Se realizaron tratamientos quirúrgicos en $79 \%$ de pacientes con enfermedad hemorroidal y en el $100 \%$ de pacientes con tumores. Conclusión: La colonoscopia es un instrumento de gran utilidad al momento de diagnóstico, seguimiento y tratamiento de las patologías Colorectales, nos permite determinar el tipo de lesión, su localización y toma 
de biopsia. Ayuda a disminuir la incidencia y morbimortalidad causada por el Cáncer Colorrectal, y así además realizar el seguimiento endoscópico de las lesiones precursoras.

Palabras claves: Colonoscopía; Cáncer Colorectal; patología colorectales; indicación de colonoscopia. 


\title{
Prevalence of Colorectal Pathologies in studies Colonoscopics: general surgery service - Central Military Hospital
}

\begin{abstract}
ABSTRAC
Introduction: Colonoscopy performs a visual exploration of the colon mucosa, among its objectives is the location of colonic lesions, obtaining biopsies for analysis, performing various therapeutic procedures or diagnosing small lesions not detectable by traditional imaging methods. The objective of this study is to determine the prevalence of Colorectal Pathologies and their characteristics in patients undergoing colonoscopies in the General Surgery Service of the Central Military Hospital of the Armed Forces during the period 2018 to 2019. Methodology: observational, descriptive, retrospective cross-sectional study. Results: 355 clinical records and colonoscopy reports were studied. Of the total, $56 \%$ corresponded to the female sex. The average age was 54 years. The most prevalent age group was 50 to 60 years. The main reason for the indication for colonoscopy was Colorectal Cancer Screninng, seen in $51 \%$ of patients, followed by lower gastrointestinal bleeding in $27 \%$ of patients. The most frequent findings in colonoscopy were Hemorrhoids in $36 \%$ of patients, followed by Polyps in $27 \%$ and Diverticula in $18 \%$. Regarding the most frequent locations: the anal canal 40\%, the sigmoid colon $32 \%$ and the descending colon $18 \%$. The histological findings, in polyps the hyperplastic type in $43 \%$ and in tumors it was colon adenocarcinoma in $82 \%$ of the cases. Surgical treatments were performed in $79 \%$ of patients with hemorrhoidal disease and in $100 \%$ of patients with tumors. Conclusion: Colonoscopy is a very useful instrument at the time of diagnosis, monitoring and treatment of Colorectal pathologies, it allows us to determine the type of lesion, its location and taking a biopsy. It helps to reduce the incidence and morbidity and mortality caused by Colorectal Cancer, and thus also perform endoscopic monitoring of precursor lesions.
\end{abstract}

Keywords: Colonoscopy; Colorectal cancer; colorectal pathology;

Artículo recibido: 25 enero 2021 Aceptado para publicación: 28 febrero2021 Correspondencia: raulcardozoarias@gmail.com Conflictos de Interés: Ninguna que declarar 


\section{INTRODUCCIÓN}

El examen endoscópico del colon se introdujo en la rutina de la práctica médica, en los inicios de la década de 1970, desde entonces la colonoscopia ha sufrido cambios importantes en cuanto no solo al diseño y tecnología de los equipos como así también los instrumentales de uso endoscópico para procedimientos terapéuticos y los diversos procedimientos técnicos para resolver patologías colonicas que anteriormente era de exclusivo resorte quirúrgico. En 1967, en la Universidad de Michigan, Overol, quien trabajaba con American Cistoscopia Makers Inc. (ACMI), presentó el primer colonoscopio de fibra óptica (De la Vega ét al 2010)

La importancia de la colonoscopia no se puede debatir. Es la exploración de referencia para diagnosticar a tiempo enfermedades de colon, como los pólipos, divertículos, enfermedades inflamatorias intestinales, tumores, fistulas y ulceras entre otras lesiones. (Ramos, 2016)

La colonoscopia es uno de los métodos más atractivos para la prevención y detección temprana del Cáncer Colorectal (CCR) ya que permite la visualización directa de toda la mucosa del colon para la detección de adenomas o CCR en etapas tempranas con la posibilidad de tomar biopsias y/o resecar lesiones durante el mismo procedimiento, es además el estudio complementario ineludible luego de hallazgos positivos en cualquiera de los otros exámenes de tamizaje. Juega un rol principal tanto en la pesquisa del CCR en población de riesgo promedio como en personas con riesgo elevado y antecedentes familiares, historias de adenomas, o CCR previos. La identificación de las lesiones preneoplásicas en la población adulta y el diagnóstico temprano de cáncer colorrectal son factores determinantes para un buen pronóstico, curación y sobrevida de los pacientes. (González et al 2010)

Este trabajo pretende describir los distintos hallazgos colonoscópicos y las características de los mismos en pacientes del Servicio de Cirugía General sometidos a colonoscopias en el Hospital Militar Central de las FFAA durante un periodo de 2 años.

\section{MATERIALES Y MÉTODOS}

Se planteo un estudio observacional, descriptivo, retrospectivo de corte transverso.

El estudio incluyo las Historias Clínicas e informes de pacientes sometidos a colonoscopias del servicio de Cirugía General del Hospital Militar Central de las FFAA de Paraguay durante el periodo 2018 a 2019. 
Criterios de inclusión: Pacientes mayores de 30 años de edad, ambos sexos, con fichas clínicas completas que hayan sido sometidos a colonoscopias durante el periodo del año 2018- 2019.

Se midieron las siguientes variables: según genero y edad, indicación de estudio, hallazgo colonoscópico, localización anatómica, hallazgos histopatológicos, tratamientos. Los datos fueron introducidos en planillas Excel y fueron analizados por medio de estadística descriptiva empleando el paquete estadístico epiinfo v.3.5.1

\section{RESULTADOS Y DISCUSIÓN}

En relación al género de las 355 historias clínicas e informes de colonoscopias estudiados, 195 pacientes correspondieron al sexo Femenino (56\%) y 160 pacientes del sexo Masculino (44\%). El promedio de edad fue de 54 años, el rango etario más frecuente fue el de 50-60 años (51\%). Al indagar cuales fueron las indicaciones de colonoscopia encontramos que el principal motivo fue Screninng de Cáncer Colorrectal (CCR) esta situación fue vista en 180 pacientes (51\%), seguido de hemorragia digestiva baja 98 pacientes (27\%), trastornos de la evacuación 66 pacientes (19\%) y por último, Enfermedad Inflamatoria Intestinal (EII) 11 pacientes (3\%).

Por su parte, en relación a los hallazgos más frecuentes en la colonoscopia fueron Hemorroides 126 pacientes (36\%), seguido de Pólipos 95 Pacientes (27\%), demás datos se observan en el gráfico 1. Hallazgos colonoscópicos de Patologías Colónicas benignas y malignas

En cuanto a las diferentes patologías colónicas tanto benignas como malignas se distribuyeron según su localización anatómica siendo las más frecuentes el canal anal $40 \%$ y el colon sigmoides $32 \%$, demás datos se muestran de la siguiente forma en el gráfico 2. Localización anatómica de Patologías Colónicas. 

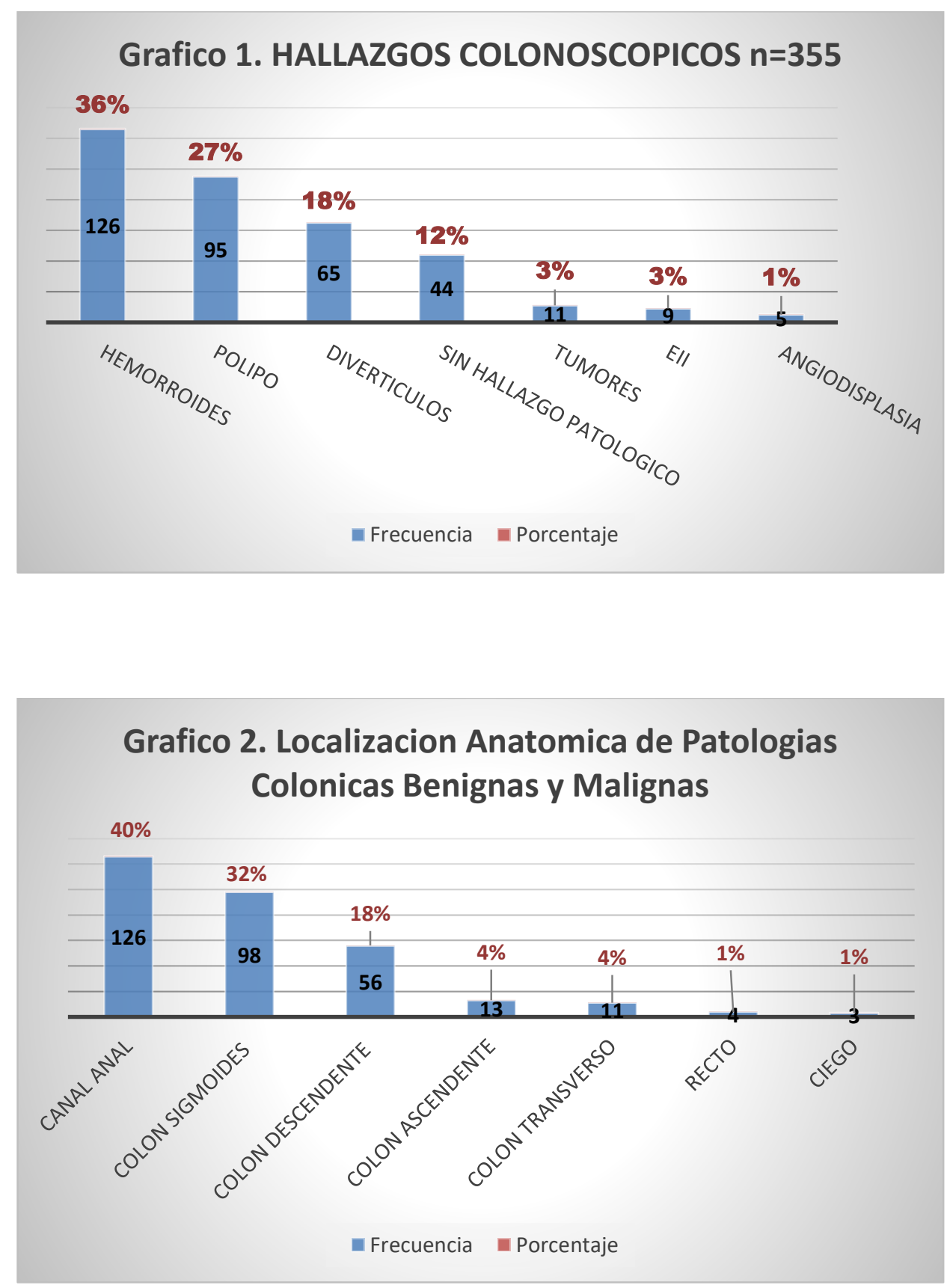

En cuanto a la localización anatómica de los pólipos colonicos encontramos un 59\% en colon sigmoideo, $28 \%$ en colon descendente, $6 \%$ en colon ascendente, $4 \%$ en colon trasverso y $3 \%$ en el ciego.

Con respecto a la localización anatómica de los divertículos encontramos un $46 \%$ en colon sigmoides, $38 \%$ en colon descendente, $8 \%$ en colon ascendente, y $8 \%$ en colon transverso. La localización anatómica de los tumores fue encontrada de la siguiente manera: $45 \%$ colon sigmoides, $27 \%$ colon descendente, $18 \%$ recto y $10 \%$ en el colon transverso. 
En relación a los hallazgos histológicos se estudiaron tanto en pólipos y tumores. En cuanto a los Pólipos colónicos ( $\mathrm{n}=95$ ); la mayoría fueron de tipo hiperplásicos en un 43\%, el resto fueron adenomas de tipo tubular 32\% y tubulovelloso 25\%. Además de los tumores $(n=11)$ en este estudio, en su mayoría fueron adenocarcinomas, $9(82 \%)$ tumores de colon y 2 (18\%) tumores de recto.

Haciendo referencia al tratamiento quirúrgico realizado en las distintas patologías reportadas, podemos acotar que de los 126 pacientes con enfermedad hemorroidal, 99 (79\%) recibieron tratamiento quirúrgico, de los cuales 1 caso fue por la técnica de longo hemorroidopexia, 60 por hemorroidectomia, 38 por macroligaduras.

En relación a los tumores $(n=11)$, los adenocarcinomas de colon $(n=9)$, fueron resueltos de forma quirúrgica en el $100 \%$ de los casos, 5 de ellos localizados en el colon sigmoides fueron realizadas sigmoidectomias con anastomosis primaria colorrectal, seguido de 3 tumores localizados en el colon descendente en los cuales se realizó colectomia izquierda con anastomosis primaria colocolonica, por ultimo 1 tumor en el transverso en el cual se realizó transversectomia también anatomosis primaria colocolonica.

Además, en los dos casos de adenocarcinoma de recto se realizó resección anterior baja con anastomosis coloanal.

En cuanto a las demás patologías reportadas, recibieron tratamiento médico y endoscópico. En el caso de los pólipos $(\mathrm{n}=95)$, fueron realizadas polipectomias en el $100 \%$ de los casos. Todos los pacientes con divertículos colónicos recibieron tratamiento médico.

\section{DISCUSION Y CONCLUSIÓN}

La Colonoscopia ha tenido un avance extraordinario durante las dos últimas décadas en la investigación sobre patologías colorectales, sea para aclarar su etiología, como para el diagnóstico temprano de patologías, así también como para escoger la terapia más adecuada y para diseñar esquemas de seguimiento y de vigilancia para los grupos de riesgo; todo orientado a mejorar el pronóstico y disminuir la morbimortalidad (Enrique ét al 2011).

En esta investigación la población estuvo constituida en su mayoría por el sexo femenino $56 \%$ (195) y el 44\% (160) del sexo masculino, lo cual coincide con la investigación presentada por Gunther A et al, (2012) en un reporte sobre colonoscopías hechas entre los años 2000 y 2010 publicado en la revista Chilena de Cirugía. 
Además concuerda con otro estudio de similar experiencia por De La Vega et al (2010) publicado en la Revista De Especialidades Médico-Quirúrgicas.

$\mathrm{Su}$ rango etario predominó en la quinta y sexta década de vida con un $51 \%$, la edad promedio fue de 54 años, correlacionándose con los resultados de un estudio observacional de Enrique, Geminiano-Martínez Eloy, et al y otros reportes similares.

Las indicaciones aceptadas para la colonoscopia fueron publicadas por The American Society for Gastrointestinal Endoscopy (ASGE) y The U.S. Multi-Society Task Force on Colon Cáncer y las dos concuerdan con el estudio.( Rex et al 2006) La principal indicación de colonoscopia en nuestro estudio fue la tamización de cáncer colorrectal $51 \%$, el cual, según recomendaciones de las distintas guías europeas y del American College of Gastroenterology, se debe realizar en personas mayores de 50 años.(Gecím et al 2016) Seguido en frecuencia por Hemorragia digestiva baja 27\%, Trastornos de la evacuación 19\%, Enfermedad Inflamatoria Intestinal (EII) 3\% (Lieberman et al 2012)

En cuanto a los hallazgos colonoscópicos, 12\% fueron colonoscopias normales sin hallazgo patológico alguno. Se reportaron hemorroides en un 36\%, según una publicación de Goodwin JS et al, sobre detección de patologías colorectales publicado en el European Medical Journal (EMJ), esta enfermedad afecta al 40-50\% de la población en algún momento de su vida y en la mayoría de los países.

Segú Goodwin et al (2011) cerca del 4\% de la población precisa de cirugía. Antes de empezar el tratamiento para las hemorroides con síntomas análogos como el sangrado, debe efectuarse la exclusión de una posible neoplasia. De nuestra muestra el 79\% fueron resueltas de forma quirúrgica y $21 \%$ requirieron tratamiento médico. Se hallaron pólipos en un $27 \%$, de los cuales en su mayoría fueron hiperplasicos en un $43 \%$, adenomas tubulares en un $32 \%$ y adenomas tubulovellosos en un $25 \%$, los cuales se localizaron mayoritariamente en el colon sigmoides en un $59 \%$ y descendente en un $28 \%$, lo que concuerda con la literatura Internacional de Espinosa (1996) y Moreno et al (2019) El adenoma es el principal precursor del Cáncer Colorrectal (CCR) y la lesión colónica más frecuentemente hallada en las colonoscopias realizadas por pesquisa. Luego de la extracción de adenomas del colon, de un tercio a la mitad de los pacientes van a presentar adenomas en colonoscopias posteriores realizadas a los tres años. Más aún, entre un 0,3 y $0,9 \%$ de ellos presentarán cáncer dentro de los 5 años. 
El objetivo principal de la vigilancia colonoscópica es prevenir el desarrollo de CCR a través de la resección de nuevos adenomas (Gualdrini 2016) Los resultados mencionados anteriormente se asemeja con otras experiencias similares, cuyas tasas de detección varían entre 10 y $25 \%$ como lo reportado por Del Valle et (2014) al publicado en una experiencia de 1 año sobre detección de lesiones del colon publicado en la Revista Scielo.

Además, se encontraron divertículos en un $18 \%$, donde la localización más frecuente fue en el colon sigmoides $46 \%$ y Colon descendente $38 \%$, lo cual coincide con lo reportado por los autores Tursi (2009) y Grande et al (2016).En su mayoría fueron asintomáticos y los casos que se presentaron por sangrado cedieron espontáneamente con tratamiento médico.

Aunque del total de pacientes analizados, un gran porcentaje presentan alguna anormalidad evidenciada en el resultado de la colonoscopia, podemos reconocer que es un estudio invasivo necesario para el diagnóstico temprano de patologías benignas y malignas colorrectales, disminuyendo la incidencia y morbimortalidad, principalmente la causada por el Cáncer Colorrectal, gracias al diagnóstico temprano, el tratamiento oportuno y el seguimiento colonoscópico de las lesiones precursoras.

Se recomienda continuar realizando estudios que permitan identificar las patologías más frecuentes que afectan el colon en los diferentes hospitales y regiones de nuestro país con el fin de generar intervenciones y estrategias masivas de tamizaje que permitan establecer un control oportuno de estas afecciones, ya que actualmente estas patologías presentan una incidencia ascendente con mayor impacto en la población mayor de 50 años.

\section{LISTA DE REFERENCIAS}

De La Vega, AR, Et Al.( 2010) "Hallazgos Endoscópicos En Estudios Colonoscópicos Realizados En El Hospital Regional 1 De Octubre Del $\quad$ ISSSTE." Revista De Especialidades Médico-Quirúrgicas;15(3): 139-143. DISPONIBLE EN https://www.medigraphic.com/pdfs/quirurgicas/rmq-2010/rmq103g.pdf

Del Valle Llufrio Pedro, Romero Bareiro Sandra Rocío, Santana Fuentes Yenia. (2014) Lesiones de colon diagnosticadas por colonoscopia en pacientes con sangre oculta positiva. Rev. Med. Electron. [Internet]; 36(1): 692-699. Disponible en: http://scielo.sld.cu/scielo.php?script=sci_arttext\&pid=S1684$18242014000700002 \& \operatorname{lng}=\mathrm{es}$. 
Espinosa Gallardo, J.M (1996). Estudio de prevalencia y morfología de pólipos intestinales en colon con y sin cáncer colorrectal. (Tesis Doctoral Inédita). Universidad de Sevilla, Sevilla. https://idus.us.es/handle/11441/40921

Enrique, G. M. E., Rolando, S. G., Oscar, M. H., Jarquín-Arremilla, A., Tafoya-Ramírez, F., \& Ángel, O. A.(2011) Endoscopic findings in 878 studies of colonoscopy. Initial experience of the Oaxacan Regional Specialist Hospital, Mexico. Evidencia Médica e Investigación en Salud, 4(2):42-47.Disponible https://www.medigraphic.com/cgi

González Huix , Monserrat Figa, Carlos (2010) Huertas Criterios de calidad que deben exigirse en la indicación y en la realización de la colonoscopiaEssential quality criteria in the indication and performance of colonoscopy [Internet]. Gastroenterología y $\quad$ Hepatología;33(1):33-42 Disponible en:https://www.sciencedirect.com/science/article/abs/pii/S0210570509004014

Gunther A, Abedrapo M, Rodrigo M, Ricardo C, José B, Berger (2012). Colonoscopia: puesta al día y reporte de 10 años de experiencia. Revista Chilena de Cirugía, 64: 306-311. Doi: https://doi.org/10.4067/ S0718-40262012000300016. Disponible: https://scielo.conicyt.cl/scielo.php?script=sci_arttext\&pid=S0718-

\section{$\underline{40262012000300016}$}

Geçim R, Fazal A, Khan A, Arshad M, Cheema M, Hayat W, Khan W, Farag A. (2016) Manejo de la enfermedad hemorroidal. Resumen descriptivo de presentaciones escogidas entre las ofrecidas en el Seminario Enfermedad venosa crónica y enfermedad hemorroidal: manejo y tratamiento, celebrada en Lisboa, Portugal, los días 23 y 24 de septiembre de Disponible : https://emj.europeanmedicalgroup.com/wp-content/uploads/sites/2/2018/02/ Manejo-de-la-Enfermedad-Hemorroidal.pdf

Goodwin JS, Singh A, Reddy N, Riall TS, Kuo YF.(2011). Uso excesivo de la colonoscopia de detección en la población de Medicare. Arch Intern Med., 171(15): 1335-1343 Disponible http://scielo.isciii.es/scielo.php?script=sci_arttext\&pid=S113001082018000500008

Gualdrini U,(2016) Pesquisa Del Cancer Colorrectal En Grupos De Riesgo Aumentado. Revista Argentina De Coloproctología;27(1): 81-126 Disponible en: https://sacp.org.ar/revista/files/PDF/27_01/capitulo_5.pdf 
Grande, G., Zulli, C., Pigò, F., Riccioni, M. E., Di Mario, F., \& Conigliaro, R. L.(2016) The role of colonoscopy in the diverticular disease. Journal of clinical gastroenterology; https://journals.lww.com/jcge/Abstract/2016/10001/The_Role_of_Colonoscopy_in _the_Diverticular.7.aspx

Lieberman DA, Rex DK, Winawer SJ et al.(2012) Guidelines for Colonoscopy Surveillance after Screening and Polypectomy: A Consensus Update by the US Multi-Society Task Force on Colorectal Cancer Gastroenterology, 143:844-857. Disponible en: https://pubmed.ncbi.nlm.nih.gov/22763141/

Moreno B. Natalia, Saavedra J. Viviana, Peters A. Tamara, von-Jentschyk R. Nicolas, Bocic A. Günther, Sanguinetti M. Antonella et al. (2019)Prevalencia de divertículos colónicos asintomáticos en población chilena como hallazgo imagenológico en tomografía de abdomen y pelvis. Rev. cir. [Internet] Jun ; 71( 3 ): 230-237. Disponible en: https://scielo.conicyt.cl/scielo.php?script=sci_arttext\&pid=S2452$45492019000300230 \& \operatorname{lng}=\mathrm{es}$.

http://dx.doi.org/10.4067/s2452$\underline{45492019000300230}$

Ramos A. ( 2016) enfermedad diverticular del colon diverticular disease of the colon [internet]. Medicine - programa de Formación Médica Continuada

Acreditado.

Disponible

en: https://www.sciencedirect.com/science/article/pii/S030454121600072X

Rex DK, Petrini JL, Baron TH, Chak A, Cohen J, Deal SE et al (2006). Quality indicators for colonoscopy. Am J Gastroenterol; 101: 873-85 Disponible https://pubmed.ncbi.nlm.nih.gov/16635231/

Tursi A, Papagrigoriadis S. (2009) Review article: the current and evolving treatment of colonic diverticular disease. Aliment Pharmacol Ther ; 30: 532-546. Doi: 10.1111/j.1365-2036.2009.04072.x

Disponible: https://pubmed.ncbi.nlm.nih.gov/19549266/ 